\title{
O DESAFIO DA AVALIAÇÃO DE EQUIPES DE ALTA CAPACITAÇÃO E AUTONOMIA: O CASO DO CREDENCIAMENTO DE DOCENTES NA PÓS-GRADUAÇÃO
}

\author{
Otavio Prospero Sanchez* \\ Lucia Helena Aponi Sanchez**
}

Recebido: jul. 2010

Aprovado: ago. 2010

\begin{abstract}
*Doutor e Mestre em Administração de Empresas pela Fundação Getulio Vargas FGV-EAESP, com pósgraduação em Finanças Corporativas pela FGV-EAESP e graduações em Engenharia de Produção e Engenharia Eletrônica. Atualmente é professor do Programa de Mestrado e Doutorado em Administração da FGV-EAESP e do Programa de Pós-Graduação em Administração da Universidade Metodista de São Paulo. E-mail: otavio.sanchez@fgv.br

**Doutora em Comunicação Social - Processos Comunicacionais, especificamente na área Organizacional pela UMESP (2006), possui Mestrado em Comunicação Científica e Tecnológica pela Universidade Metodista de São Paulo (1997). Graduada em Comunicação Social, sendo Bacharel em Relações Públicas pelo Instituto Metodista de Ensino Superior (1983). Atualmente é Presidente do Conselho de Ensino a Distância da Universidade Bandeirante de São Paulo, atuando como Gestora do Núcleo de Educação a Distância. E-mail: lucia.sanchez@terra.com.br
\end{abstract}

Resumo: A avaliação de equipes de alta capacitação e autonomia representa tradicionalmente um grande desafio para os gestores das organizações. Em particular, quando a estratégia da organização demanda o realinhamento da equipe, o sucesso da iniciativa pode não ser facilmente atingido mesmo que os gestores disponham de alto poder coercitivo, uma vez que os processos são executados com autonomia pela equipe, tornando difícil a efetiva monitorização e controle, dificuldade que se potencializa pela alta capacitação dos membros da equipe. Adicionalmente, o emprego do poder coercitivo pode ter o reflexo negativo da queda do desempenho pelas reações individuais ou articuladas, típicas da resistência à mudança. Consequentemente, o comprometimento dos membros de uma equipe de alta capacitação e autonomia pode requerer o uso de técnicas que atendam a necessidades individuais como a manutenção do nível de reconhecimento prévio junto ao grupo. Este trabalho trata da apresentação de técnica que simultaneamente permite objetivar o alinhamento da equipe e promover o reforço de percepções importantes para o comprometimento de indivíduos com esse perfil tais como reputação, autonomia e estabilidade, que contribuem para o sucesso em ações que alinhem indivíduos aos objetivos da organização, reduzindo a resistência à mudança. $\mathrm{O}$ trabalho discorre sobre os aspectos teóricos da tensão gerada pela necessidade de alinhamento de uma equipe com essas características e as implicações no nível do indivíduo, apresentando em seguida uma proposta de condução dessa situação conduzida com base no método de pesquisa-ação em um programa de pós-graduação stricto-sensu no Brasil.

Palavras-chave: Alinhamento de equipes. Equipes autônomas. Gestão de equipes altamente capacitadas.

\section{THE CHALLENGE OF EVALUATING TEAMS OF HIGH CAPACITY AND AUTONOMY: THE CASE OF ACCREDITATION OF PROFESSORS IN GRADUATE PROGRAMS}

Abstract: The evaluation of highly qualified and autonomous teams traditionally represents a major challenge for managers of organizations. Particularly when the strategy of the organization demands the realignment of the team, the success of the initiative may not be easily achieved even if the managers possess high coercive power, since the processes are executed independently by the 
team, making it difficult to effectively monitor and control, a difficulty which is intensified by the high qualification of the team members. Furthermore, the use of coercive power may have the negative effect of the decrease in performance by individual or articulated reactions, typical of the resistance to change. Consequently, the commitment of members of a highly qualified and autonomous team may require the use of techniques that meet the individual needs such as maintaining the level of prior recognition by the group. This work presents a technique that allows to simultaneously aim for the alignment of the team and promote the reinforcement of perceptions important to the commitment of individuals with this profile, such as reputation, independence and stability, which contribute to the success of actions that align individuals to the objectives of the organization, reducing resistance to change. The paper addresses the theoretical aspects of the tension generated by the need to align a team with these characteristics and implications at the individual level, then presenting a proposal to conduct this situation based on the research action method in a stricto sensu graduate program in Brazil.

Key words: Team alignment. Autonomous teams. Management of highly qualified teams.

\section{INTRODUÇÃO}

Do ponto de vista das teorias econômicas aplicadas ao comportamento dos indivíduos, podemos afirmar que, em geral, somos resistentes a mudanças. Mudanças tendem a nos colocar em situações em que a percepção de risco aumenta, dada à incerteza associada ao futuro. Quando nos confrontamos com uma mudança, o futuro passa a ser percebido como mais incerto, o que pode desencadear três reações: aversão ao risco, apreciação ao risco ou indiferença (MILGROM; ROBERTS, 1992).

A Psicologia também consagra o comportamento de aversão a riscos como associado à ânsia do individuo de satisfazer a necessidade de prazer ou felicidade, em oposição ao sofrimento (NG, 2003). De fato, conceitos advindos de teorias da Economia e Psicologia vêm convergindo em direção à tentativa de proposição de um conjunto explicativo sobre o comportamento humano em situações conflituosas submetidas a risco (GLIMCHER; RUSTICHINI, 2004).

Em ambas as abordagens, entretanto, os indivíduos são entendidos como seres que ordenam suas preferências de maneira racional, dadas as suas próprias referências de racionalidade e escala de valores. Assim, os indivíduos tendem a ser avessos a mudanças porque, do ponto de vista psicológico, isso pode representar um questionamento a seu conjunto de respostas para problemas dados em situações rotineiras, que já estão anteriormente resolvidos, ameaçando o estado de felicidade gerado pela realização da sensação de proteção. Do ponto de vista econômico, a aversão à mudança ainda depende da matriz de pagamentos percebida que as várias novas alternativas possíveis podem gerar, e sua ordenação pela percepção de risco associadas a elas, co- 
nhecido pelo conceito chamado utilidade marginal. (PINDYCK; RUBINFELD, 2006)

Para ambos os casos, os indivíduos priorizam as alternativas e valoram suas percepções tendo como base um conjunto de padrões pessoais que são individualizados, mas influenciados pela construção coletiva do meio social pela qual o indivíduo se referencia. Tal construção é resposta racional à presença de experiências emocionais positivas e à ausência de experiências emocionais negativas no contexto da socialização (DIENER, 2000; HALLER; HADLER, 2006).

Estudos multinível que relacionam indivíduos e grupos fornecem melhor explicação sobre os conceitos de integração e pertença nos quais os indivíduos se referenciam. Indivíduos são estimulados por uma necessidade de pertencer a um determinado grupo que os reconheça e valorize, por meio de fatores que são associados à geração de bem-estar afetivo (GAVIN; MASON, 2004). Pessoas apresentam a necessidade de formar e manter relacionamentos (BAUMEISTER; LEARY, 1995) em uma visão não apenas afiliativa objetivando a aceitação do grupo, mas também visando o estabelecimento de relacionamentos contínuos e de qualidade que geram satisfação (DE CREMER; BLADER, 2006).

Para obter aceitação, o indivíduo se submete a um esquema de confirmação ou rejeição das ações individuais que, embora também influenciado por suas próprias opiniões de ordenação de valores, acaba por tornar-se uma estrutura socialmente construída que enfatiza o que é aceito e considerado adequado pelo grupo, desestimulando o que ocorrer em desacordo com tais conceitos (GIDDENS, 1991).

Há grupos em que é levada a influência dos indivíduos no processo de definição da estrutura de valores. Particularmente, quando indivíduos têm lugar no processo formal de decisão organizacional por meio de representação colegiada, tendo em vista que os processos desempenhados têm como requisitos a alta capacitação e autonomia, pode haver uma tendência de grande estabilidade de padrões e critérios de reforço. A tendência disso ocorrer se deve ao fato de a estrutura de reforço sofrer ciclos de aperfeiçoamentos sucessivos que confirmam aspectos aceitos pelo grupo, rechaçando os desvios, em um processo de convergência contínua a valores únicos.

Essa característica peculiar desse tipo de grupo torna particularmente difícil a alteração coercitiva dos processos, ainda que os gestores possuam alto poder formal, já que emoções negativas podem contribuir para que indivíduos percam a disposição para enfrentar situações adversas, a ficarem mais pro- 
pensos aos sintomas do estresse e a apresentarem dificuldades de desenvolver relações sociais positivas. Como conseqüência, o clima organizacional pode piorar e a produtividade reduzir-se (GAVIN; MASON, 2004).

Este trabalho visa indicar como a aplicação de uma técnica apropriada pode ajudar no estabelecimento de novos comportamentos e na aceitação de estruturas de valores diversas daquelas estabelecidas pelas equipes, quando formadas por indivíduos onde a alta capacitação e autonomia são fundamentais para os resultados.

\section{DELIMITAÇÃO DO PROBLEMA: CREDENCIAMENTO DE DOCENTES PERMANENTES}

Os sistemas de informações utilizados para avaliar os Programas de PósGraduação Stricto-Sensu no Brasil estão evoluindo. Nos últimos anos, o número de Programas de Pós-Graduação no Brasil aumentou significativamente, e o nível de qualidade desses programas também tem evoluído.

Estabeleceu-se, desde 1976 um conjunto de mecanismos que visa primordialmente a estimular que os Programas ampliem a geração de conhecimento e a formação de recursos humanos, e que vem evoluindo em sua sistemática de avaliação. Como proxy dessa evolução, os Conselhos Técnicos Científicos da Áreas estabeleceram um conjunto de indicadores, em sua maioria objetivos, que operam tendo como base a publicação alcançada pelo Programa, sua quantidade e qualidade.

Embora tendo recebido críticas (SPAGNOLO; SOUZA, 2004) sob o argumento de que seria excessivamente quantitativo, esse novo cenário, que é muito diferente da dinâmica que a Pós-Graduação brasileira viveu até então, tem estimulado os Programas a uma busca sistemática pelo seu aperfeiçoamento.

Certas evoluções tem sido meritórias enquanto que outras tem sido caracterizadas por uma reação de curto-prazo que perde de vista os objetivos maiores de melhoria do sistema de Pós-Graduação e desenvolvimento do país no sua capacitação de lidar com o conhecimento.

O próprio sistema vem sendo aprimorado em função da análise de seus resultados (SPAGNOLO; SOUZA, 2004; CAPES, 2008). Uma das orientações recentes da CAPES sobre os Programas é de que estes implementem um sistema efetivo de credenciamento de docentes pertencentes ao Núcleo Docente Permanente - NDP, que garanta um conjunto mínimo de regras para a sua afiliação e desafiliação a um Programa. Dentre outros objetivos, essa requisito 
de formalização pode reduzir a possibilidade de que a gestão dos Programas seja estimulada a otimizar, apenas pela perspectiva de curto-prazo, a alocação de docentes em função de sua publicação efetivada ou por se realizar.

Assim, não havendo um mecanismo efetivo de indicação de professores NDP, Programas poderiam ser geridos pelo viés de potencializar o resultado numérico das publicações, em detrimento da estabilidade do quadro de docentes permanentes. Como todo Programa de Pós-Graduação está afeto a uma Faculdade, e esta geralmente conta com muitos docentes disponíveis em seus quadros, haveria a possibilidade de que a gestão do Programa estabelecesse uma espécie de rodízio de docentes permanentes, tomando como critério de afiliação apenas o desempenho em iminente publicação relevante.

Essa prática, se implementada, poderia ser altamente perniciosa para o sistema de avaliação porque premiaria com boas avaliações um Programa que, embora obtendo resultados positivos ainda assim estariam contribuindo pouco para o efetivo desenvolvimento de um núcleo de conhecimento.

Assim, é uma boa prática que os Programas implementem sistemas no sentido de estabelecer que o credenciamento docente se dê por meio de um procedimento formal, estável e que leve em conta critérios claros e previamente estabelecidos.

Entretanto, o processo de credenciamento usualmente requer o atendimento de interesses de vários outros participantes além da própria CAPES. Alguns participantes que têm interesse na maneira como a identificação de professores mais adequados será feita, são:

1) Os alunos, que desejam que os melhores professores estejam disponiveis no curso, assim como apreciam ter contato com conhecimentos atualizados, usualmente gerados por meio de pesquisas conduzidas por professores competentes nessa atividade;

2) A Instituição, representada pela gestão do Programa, que tem interesse em sinalizar para a CAPES e alunos que há forte estímulo para que apenas professores muito competentes permaneçam em seus quadros de docentes permanentes;

3) Os professores, que serão efetivamente confirmados ou não como NDPs, e que desejam manter a estrutura vigente, tendo em vista a sua aversão ao risco representado pela nova dinâmica que adiciona elementos de incerteza e não lhes permite identificar claramente qual sua posição no futuro; 
Tal caracterização sinaliza para um aumento da complexidade do problema, por adicionar critérios diversos, próprios de cada interessado, que muitas vezes podem ser divergentes entre si.

De um lado, os resultados do Programa na geração de conhecimento, medidos indicadores da quantidade e qualidade da publicação alcançada e objetivo maior dos sistemas de avaliação da CAPES, dependem fortemente da equipe de professores NDPs, de sua criatividade e competência na execução da atividade de pesquisa. Isso requer a ênfase, por parte da gestão do Programa, na autonomia e capacitação do docente, atributos altamente valorizados pela academia. Também são consagrados no ambiente acadêmico, processos de decisão colegiada, onde cada professor participa das decisões do Programa, e a coordenação é exercida em um regime de rodízio entre professores NDPs, arranjo que favorece a decisões sejam tomadas tendo como base na não prevalência de uma abordagem sobre outras, em alinhamento com uma visão científica própria do ambiente em que se insere a atividade.

A demanda da CAPES, entretanto, indica que um sistema de avaliação deva ser implementado. Tal sistema, entretanto, deve ser proposto e decidido pelo colegiado, que é composto por professores a serem submetidos à avaliação. Em razão da aversão individual ao risco e da interconexão existente entre o indivíduo e a estrutura de valores do grupo, pode-se supor que, as decisões emanadas do colegiado podem supervalorizar elementos de suas próprias demandas como professores, em detrimento de outros aspectos, como os relativos à nova sistemática de produtividade indicada pela CAPES.

Entretanto, impor coercitivamente um conjunto de critérios pela via do poder formal da função de Coordenação, tende a contrariar os indivíduos em dimensões que vão além da simples opinião sobre este ou aquele item, mas remete à experiência emocional negativa de desconfirmar valores típicos da academia como a autonomia, liberdade de expressão e reputação. Além disso, a referência que o indivíduo faz em relação aos critérios que lhe permitem a aceitação no grupo pode dificultar, ou mesmo inviabilizar, a implantação de novos procedimentos que visem modificar os comportamentos do grupo, o que pode torná-la uma diretriz que não se transforma em ação efetiva (FISS; ZAJAC, 2006).

O problema se configura, então, na demanda pela reorientação da prática de um grupo altamente capacitado e autônomo, em certa dimensão auto-gerido em função da tradição de decisões colegiadas próprias da academia. Essa reorientação é configurada especialmente pela diretriz do órgão regulador dos Programas de Pós-graduação, a CAPES, que deve ser implementada visando 
o efetivo fomento ao desenvolvimento do conhecimento, por meio da execução de pesquisas originais. Essa implementação não deve, entretanto, configurar uma percepção excessiva de risco para os professores, ou o completo rompimento com os valores estabelecidos na cultura organizacional, de forma a não comprometer seu desempenho (PARKER et al., 2003).

\section{ABORDAGEM METODOLÓGICA}

Neste trabalho, adotamos a abordagem da pesquisa-ação, que pode ser considerada um tipo de estudo de caso, com a diferença que o pesquisador deixa de ser um observador independente para tornar-se um participante, e o processo de mudança seu objeto de pesquisa. Portanto, o pesquisador tem simultaneamente o objetivo de agir para solucionar um problema e contribuir para um conjunto de conceitos que complementem a teoria (BENBASAT; GOLDSTEIN; MEAD, 1987).

A pesquisa-ação é uma concepção de pesquisa e intervenção em situações sociais, junto a atores significativos, em processos de mudança, onde a participação do pesquisador visa sucessivamente descrever o problema com base em verbalizações dos diferentes autores em suas próprias linguagens, e intervir por meio dos conhecimentos derivados das inferências que são usados para a elaboração das ações (THIOLLENT, 1997).

Um aspecto importante na pesquisa-ação é, diferentemente de encontrar uma solução ótima, como em outros métodos, conseguir que a mudança seja feita, relatando a aplicação da teoria e também a resistência à aplicação de determinada técnica. Deve ser aplicado por meio de uma seqüência de passos que são: (1) Diagnóstico: Definir o problema; (2) Planejamento: considerar as alternativas de ação para resolver o problema; (3) Atuação: executar a ação definida; (4) Avaliação: Estudar as conseqüencias da ação; (5) Relato: documentar e relatar o que foi aprendido (SUSMAN; EVERED, 1978).

A abordagem da pesquisa-ação é particularmente interessante quando se deseja compreender o fenômeno a partir da ótica dos participantes e da superação dos obstáculos encontrados por eles para a mudança pretendida. Embora o conhecimento gerado seja de generalização parcial uma vez que está fortemente ligado ao contexto da pesquisa que a origina, sua contribuição pode ser particularmente atrativa nos seguintes contextos (THIOLLENT, 1997):

- Quando há a possibilidade de coletar informações originais, em situações e atores no mundo real; 
- Quando pode haver divergência entre o saber formal e o saber informal nas tentativas de solução dos problemas;

- Quando se deseja conhecer as regras práticas na resolução de problemas e planejamento de ações;

- Quando se considera relevante conhecer os resultados positivos e negativos das ações colocadas em prática.

\section{CARACTERIZAÇÃO DO CASO E PROPOSTA DE AÇÃO}

O caso é um Programa de Pós-Graduação em Administração, de uma Universidade do Estado de São Paulo. O Programa completou 3 anos em 2007 e obteve conceito 4 na avaliação da comissão de área de Administração na primeira avaliação trienal da qual participou, em 2006. A Faculdade a que se filia o Programa conta com cerca de 4000 alunos em seus cursos de graduação, distribuídos em suas diversas variantes da área principal de Administração. A Faculdade está inserida em uma Universidade de médio porte, com 47 cursos de graduação, 57 cursos de Pós-Graduação Lato-sensu e 8 programa de PósGraduação Strito-sensu, com um total de cerca de 15.000 alunos.

O problema inicial consistiu de obter do grupo de decisores a definição de um construto que pudesse descrever a característica de credenciamento docente permanente em um Programa de Pós-graduação. O contexto identificado como obstáculos ao processo de definição de um conjunto de atributos pertinentes ao conceito de docente credenciável, são listados a seguir:

1) A estratégia de criação de um instrumento é exógena, criada em função do objetivo de se atender a exigências do órgão regulador da atividade;

2) O sistema de avaliação que se objetivava definir serviria para avaliar os próprios decisores;

3) Os objetivos dos vários interessados: professores, gestores e alunos não são completamente alinhados entre si;

4) Os decisores envolvidos apresentam perfil de alta autonomia, capacitação e reputação que os torna resistentes a mudanças em relação aos valores e procedimentos existentes, e percebem risco na modificação dos procedimentos existentes; 
5) As definições da estrutura atual de valores do grupo são resultados de repetidas rodadas de interação, o que faz com que os indivíduos percebam incentivos em conduzir negociações explícitas ou tácitas sobre aspectos individuais e relevantes entre si;

6) O grupo é pequeno, o que torna a fácil e efetiva a sinalização entre participantes, assim como cada integrante é muito influente no processo de formação da estrutura de valores do grupo;

7) O processo formal de decisão do grupo é democrático, por meio de colegiado; estilo esse típico da academia;

8) Os processos que se quer fomentar pela instituição da avaliação referem-se ao desenvolvimento de conhecimento, e são fortemente dependentes das do comportamento e das posteriores ações individuais dos membros dos grupos, que são produzidas de uma forma autônoma e criativa;

9) Diferentes individuos têm diferentes percepções para a contribuição dos atributos na composição da avaliação, e percebem de maneira diferente os riscos individuais da inclusão de cada item na avaliação.

Considerando a necessidade de engajamento dos participantes no processo decisório e de garantir o comprometimento individual posterior à implementação do processo emergente, a proposta de ação escolhida foi a de implementar uma técnica multicritério de composição da decisão, operacionalizada pelo método Analytic Hierarquical Process - AHP, que foi disponibilizado por meio do software ExpertChoice 11.5 (EXPERTCHOICE, 2008) O AHP foi desenvolvido por Thomas Saaty e vem sendo crescentemente aplicado a situações onde os processos de decisão são complexos (SAATY, 1977).

O AHP consiste de um processo de facilitação para a tomada de decisão em situações complexas, com múltiplos decisores e objetivos conflitantes (SAATY, 1990). Em particular, um dos atributos que melhor indicaram sua aplicação neste caso, foi a possibilidade de se contribuir para dois aspectos, considerados fundamentais para a continuidade do processo: (1) a produção de um consenso sobre os atributos a serem incluídos e sua ordenação relativa (ZAHEDI, 1986) e (2) o engajamento dos participantes na solução final e na sua legitimação .

O método AHP usualmente é bastante útil quando a decisão envolve a análise de várias alternativas diferentes entre si, sendo que não ocorre a dominân- 
cia de nenhuma alternativa, isto é, não existe alternativa que é melhor em todos os aspectos a serem avaliados, configurada por uma opinião dominante ou hegemônica. O que ocorre na grande maioria dos casos é que as alternativas, neste caso os atributos a serem incluídos no construto de avaliação, alternem entre si as preferências dos participantes. A questão é, então, o que fazer para computar as alternativas e como classificá-las de uma forma que possa ser produzido um consenso no julgamento sobre qual a melhor alternativa (HARKER; VARGAS, 1987).

As falhas de percepção dos decisores aumentam as dificuldades do julgamento das alternativas. Isso ocorre, em geral, pela assimetria de informações, situação em que o decisor não tem todos os detalhes necessários para produzir um julgamento completo e perfeito sobre o que precisa ser decidido. Quando isso acontece, fatores de subjetividade adicionais podem passar a fazer parte do julgamento do decisor, na medida em que faltam informações que permitam o julgamento adequado.

Outra dificuldade para a tomada de decisão sobre as alternativas envolve a questão da percepção do risco. A teoria da agência explica que os decisores são, via de regra, muito mais avessos a risco do que seria de se esperar tendo em vista as decisões que tomam ao computarem na opinião sobre os fatores de decisão os aspectos pessoais como as conseqüências que um mau resultado podem ter sobre sua carreira, em lugar de emitirem uma opinião desinteressada sobre o atributo. Ao considerarem os aspectos pessoais adicionalmente aos aspectos envolvidos na decisão, os decisores modificam os prêmios ou penalidades da matriz de resultados das decisões, desequilibrando-a (MILGROM; ROBERTS, 1992).

Assim, aspectos pessoais como dificuldade de avaliação e percepção ao risco, podem interferir na maneira com a decisão é tomada. Por essa razão, em particular quando o assunto adquire um elevado grau de complexidade, as decisões centralizadas podem comprometer demasiadamente os resultados.

Um aspecto muito importante da decisão colegiada diz respeito ao comprometimento resultante desse tipo de decisão, em comparação à uma decisão coercitiva. Especialmente nas situações em que o futuro não é totalmente previsível, o resultado da implementação pode ser altamente dependente do envolvimento pessoal dos gestores ao longo do processo. Assim, o comprometimento da estrutura organizacional resultante de uma decisão tomada de forma colegiada tende a ser um facilitador da solução dos problemas que surgirão durante o processo de implementação. Em contrapartida às vantagens mencionadas, a decisão colegiada envolve múltiplas percepções que dificultam 
a tomada de decisão. Em geral, a maior dificuldade refere-se à produção do consenso ou à aceitação dos critérios de decisão previamente à tomada de decisão. O desafio é uniformizar o entendimento das alternativas e do processo de decisão ao longo da equipe de decisores, assim como produzir a aceitação dos critérios de classificação das alternativas.

\section{DESCRIÇÃO DO MÉTODO E DA SUA APLICAÇÃO}

Por ser um modelo hierárquico, no método AHP a estruturação da decisão se dá por meio de uma árvore ou hierarquia da decisão, antes que o processo de ordenação de alternativas seja encaminhado. Isso faz com que os decisores tenham que compreender e concordar com os procedimentos de decisão antes das alternativas serem avaliadas, o que facilita o consenso por reduzir os atritos de julgamento na fase inicial (SAATY, 1990).

O AHP usa a abordagem de média ponderada de pesos para os atributos da decisão. Essas ponderações de atributos são feitas de forma considerada mais consistente que a simples atribuição de valores para cada alternativa porque as comparações são feitas aos pares. Há vários estudos que indicam que os indivíduos não são bons juízes para elicitar valores absolutos de variáveis mas, em geral, são bastante efetivos em ordenar variáveis (FISCHHOFF, 1991). Dessa maneira, o método AHP baseia-se na abordagem de comparação pareada de valores de atributos. O procedimento completo consiste dos seguintes passos:

Definição dos stakeholders da decisão: Deve-se cuidar para que seja criada uma representação hierárquica da decisão que deve ser feita de maneira a envolver os vários interessados ou participantes da decisão representada pela variável que definirá o credenciamento docente, também conhecidos por stakeholders da decisão. Nessa etapa são escolhidos os participantes da decisão, que neste caso representaram todos os docentes permanentes, que são membros do colegiado de Programa, o Coordenador do Programa, o Diretor da Faculdade a que o Programa se afilia, o Pró-Reitor de Pós-Graduação e o representante do corpo discente. O objetivo dessa escolha consistiu de envolver as diversas esferas de atuação e interessados, de forma a contemplar a diferentes visões sobre os requisitos necessários para o credenciamento docente.

Uma prática corrente na aplicação do método consiste de se criar um mapa de decisão onde se busca identificar os decisores e seus perfis básicos na decisão. Podem-se classificar os decisores sob o ponto de vista de seu papel na decisão, como por exemplo, papel decisor, papel aconselhador, ou ainda decisores que apresentem poder de veto. Esse mapeamento prévio é de grande im- 
portância dado que os participantes criam expectativas sobre a sua influência na decisão final, que pode não corresponder, na exata proporção, à realidade do processo decisório.

Nessa fase, a lista de decisores deve ser criada e devem-se estabelecer quais pesos na decisão cada decisor terá. Por exemplo, a decisão pode ser democrática, com 1 voto atribuído a cada decisor da lista, ou pode ser ponderada de acordo com aspectos organizacionais ou culturais. É importante que esses pesos sejam antecipadamente conhecidos pela equipe que está produzindo a decisão para que as expectativas quanto aos papéis na decisão sejam previamente conhecidos, o que tende a melhorar o comprometimento com a decisão final.

Na situação descrita neste trabalho, os pesos relativos da decisão corresponderam a 1 (hum) para cada docente, que totalizaram oito indivíduos, 1 (hum) para o representante discente e 2 (dois) para o voto da Pró-reitoria, da Diretoria, da Coordenação.

Criação de uma representação hierárquica dos atributos de decisão: Em um primeiro momento, o objetivo é a criação de uma lista de decisores e definição de seus papéis. Nesse momento, ainda não se tem claros todos os atributos que serão utilizados na decisão, mas pode-se ter uma idéia bem definida da natureza desses atributos. Em seguida, procede-se à criação da hierarquia de atributos de decisão que será a base do método. Nesse momento, usualmente procura-se listar todos os possíveis atributos a serem considerados na decisão. Não se recomenda que, a priori, a hierarquia seja criada concomitantemente à atribuição de valores relativos para os atributos. Ao contrário, os atributos devem ser listados independentemente de sua importância relativa para que todos os aspectos possam ser considerados. Posteriormente, a própria aplicação do método AHP se encarregará de excluir os atributos que tenham pouca importância de acordo com a opinião majoritária dos decisores. Nessa fase, costuma-se aplicar a técnica de brain storming para a geração de uma lista compreensiva de atributos a serem considerados.

É considerada uma boa prática construir a hierarquia da decisão por meio de agrupamentos de atributos em conjuntos de tamanho entre 5 e $9(7 \pm 2)$ elementos. Esse número (7) é considerado o ideal na literatura clássica sobre o volume de informação com o qual os decisores podem lidar adequadamente (MILLER, 1956). Assim, hierarquias representativas da decisão devem ser organizadas de maneira a que tenham o tamanho ideal para a facilitação do processo de decisão, ainda em especial quando se trata de decisões colegiadas.

$\mathrm{O}$ apêndice A ilustra o resultado do procedimento de brain-storming realizado entre os participantes, com o objetivo de colher as sugestões de todos, 
independentemente da ponderação relativa que cada item pudesse ter ao final. Destaque-se que este é um dos principais elementos que permitem o comprometimento dos envolvidos, já que todos podem, democraticamente, sugerir aspectos que entendam como adequados, independente de juízo de valor.

Desenvolvimento das ponderações para cada critério: Essa fase é cumprida por meio do procedimento de criação de uma matriz de critérios que permite sua comparação entre pares. Cada participante deve atribuir conceitos de comparação entre os pares por meio de notas que melhor descrevam essas preferências, conforme a quadro abaixo.

\begin{tabular}{|c|l|}
\hline Nota relativa & Descrição \\
\hline 1 & Preferência idêntica \\
\hline 3 & Preferência moderada \\
\hline 5 & Preferência forte \\
\hline 7 & Preferência muito forte \\
\hline 9 & Preferência extremamente forte \\
\hline
\end{tabular}

Quadro 1 - Estrutura de comparações de preferências entre pares

Fonte: (SAATY, 1990)

Assim, supondo que em certo nível da hierarquia da decisão, $\mathrm{n}$ sejam os atributos envolvidos. Assim, o conjunto de atributos de mesmo nível na hierarquia será dado por $\mathrm{x} 1, \mathrm{x} 2 \ldots \mathrm{xn}$, Os julgamentos quantificados aos pares serão representados por uma matriz A ( $\mathrm{n} \times \mathrm{n})$, onde:

$$
A=\left(a_{i j}\right)(\text { para i, } \mathrm{j}=1,2, \ldots, \mathrm{n})
$$

Por exemplo, suponhamos que estejamos avaliando 3 critérios: "Titulação", "Visibilidade na Comunidade Acadêmica " e "Titulação". Teremos, como resultado dessas comparações aos pares, uma matriz $\mathrm{A}_{(3: 3),}$ onde:

- Se $a_{i j}=k$, então $a_{j i}=1 / k ; a \neq 0$

- Se $x_{i}$ é julgado como de preferência idêntica em relação $x_{j}$, então $\mathrm{a}_{\mathrm{ij}}=1$ e $\mathrm{a}_{\mathrm{ji}}=1 / 1$ 
Assim, a matriz exemplificada teria a seguinte forma geral:

$$
\mathrm{A}=\left(\begin{array}{cccc}
1 & a_{12} & \cdots & a_{1 n} \\
1 / a_{12} & 1 & \cdots & a_{2 n} \\
\vdots & \vdots & \cdots & \vdots \\
1 / a_{1 n} & 1 / a_{2 n} & \cdots & 1
\end{array}\right)
$$

A forma específica da matriz do nosso exemplo, supondo que as relações de preferências entre os três atributos fossem "Habilidade em Pesquisa e Publicação" $\succ$ "Visibilidade na Comunidade Acadêmica " $\succ \succ$ "Titulação"; (onde: $\succ$ : significa "preferível a" e $\succ \succ$ : significa "muito preferível a") ficaria, então:

\begin{tabular}{l|ccc} 
& $\begin{array}{c}\text { Habilidade em } \\
\text { Pesquisa } \\
\text { e Publicação }\end{array}$ & $\begin{array}{c}\text { Visibilidade na } \\
\text { Comunidade } \\
\text { Acadêmica }\end{array}$ & Titulação \\
\hline $\begin{array}{l}\text { Habilidade em Pesquisa } \\
\text { e Publicação }\end{array}$ & 1 & 3 & 9 \\
$\begin{array}{l}\text { Visibilidade na Comunidade } \\
\text { Acadêmica }\end{array}$ & $1 / 3$ & 1 & 7 \\
Titulação & $1 / 9$ & $1 / 7$ & 1 \\
\hline
\end{tabular}

Cada matriz resultante da comparação de atributos deve ser analisada com base em sua normalização para que seja possível a padronização subseqüente das alternativas. Para isso, assume-se que $W$ como a somatória dos pesos individuais. Matematicamente, temos:

$$
W_{j}=\sum_{i=1}^{n} a_{i j} \quad(\mathrm{j}=1,2, \ldots, \mathrm{n})
$$

\begin{tabular}{|c|c|c|c|}
\hline & $\begin{array}{l}\text { Habilidade em } \\
\text { Pesquisa e } \\
\text { Publicação }\end{array}$ & $\begin{array}{l}\text { Visibilidade na } \\
\text { Comunidade } \\
\text { Acadêmica }\end{array}$ & Titulação \\
\hline $\begin{array}{l}\text { Habilidade em Pesquisa } \\
\text { e Publicação }\end{array}$ & 1 & 3 & 9 \\
\hline $\begin{array}{l}\text { Visibilidade na Comunidade } \\
\text { Acadêmica }\end{array}$ & $1 / 3$ & 1 & 7 \\
\hline Titulação & $1 / 9$ & $1 / 7$ & 1 \\
\hline W & 1,44 & 4,14 & 17,0 \\
\hline
\end{tabular}

Em nosso exemplo, na fase de normalização, teríamos: 
A seguir, calcula-se o valor normalizado, dado por:

$$
\mathrm{A}=\left(\begin{array}{cccc}
w_{1} / W_{1} & w_{1} / W_{2} & \cdots & w_{1} / W_{n} \\
w_{2} / W_{1} & w_{2} / W_{2} & \cdots & w_{2} / W_{n} \\
\vdots & \vdots & \cdots & \vdots \\
w_{n} / W_{1} & w_{n} / W_{2} & \cdots & w_{2} / W_{n}
\end{array}\right)
$$

Isso resultaria, em nosso exemplo, uma matriz normalizada conforme abaixo, onde se pode também calcular o peso relativo de cada atributo, dado por:

$$
P_{i}=\frac{1}{n} \sum_{j=1}^{n} a_{i j}(\mathrm{i}=1,2, \ldots, \mathrm{n})
$$

\begin{tabular}{|c|c|c|c|c|}
\hline & $\begin{array}{l}\text { Habilidade em } \\
\text { Pesquisa e } \\
\text { Publicação }\end{array}$ & $\begin{array}{l}\text { Visibilidade na } \\
\text { Comunidade } \\
\text { Acadêmica }\end{array}$ & Titulação & PESO RELATIVO DO ATRIBUTO \\
\hline $\begin{array}{l}\text { Habilidade em } \\
\text { Pesquisa e } \\
\text { Publicação }\end{array}$ & $\begin{array}{l}1 / 1,44= \\
0,692\end{array}$ & $\begin{array}{l}3 / 4,14= \\
0,724\end{array}$ & $\begin{array}{l}9 / 17= \\
0,529\end{array}$ & $P_{1}=\frac{0,692+0,724+0,529}{3}=0,649$ \\
\hline $\begin{array}{l}\text { Visibilidade na } \\
\text { Comunidade } \\
\text { Acadêmica }\end{array}$ & $\begin{array}{l}0,33 / 1,44= \\
0,231\end{array}$ & $\begin{array}{l}1 / 4,14= \\
0,241\end{array}$ & $\begin{array}{l}7 / 17= \\
0,412\end{array}$ & $P_{2}=\frac{0,231+0,241+0,412}{3}=0,295$ \\
\hline Titulação & $\begin{array}{l}0,11 / 1,44= \\
0,077\end{array}$ & $\begin{array}{l}0,14 / 4,14= \\
0,034\end{array}$ & $\begin{array}{l}1 / 17= \\
0,059\end{array}$ & $P_{3}=\frac{0,077+0,034+0,059}{3}=0,057$ \\
\hline
\end{tabular}

Tendo encontrado os pesos relativos dos atributos em um mesmo nível na hierarquia da decisão, pode-se, então, efetuar o mesmo procedimento para cada nível da hierarquia, de maneira a encontrar os diversos pesos componentes da decisão como um todo. 
Cada matriz resultante da comparação de atributos deve ser analisada para se verificar a consistência das comparações entre pares e a classificação correspondente. Isso decorre do fato do decisor estabelecer suas avaliações aos pares, sem ter uma visão do conjunto, de modo que inconsistências podem surgir, em especial quando a dimensão da matriz de comparação aumenta. $\mathrm{O}$ processo sugere que uma razão de inconsistência deva ser calculada e que seu resultado deve ser menor que 0,1 . Se o resultado for superior a esse valor, deveria provocar uma revisão por parte do decisor, conforme sugestão de Saaty (SAATY, 1990).

Para se avaliar, a cada matriz de comparação se a consistência dos julgamentos é aceitável ou se deve ser reavaliada, deve-se calcular:

$$
\text { Índice de Consistência }(C I)=(\lambda-\mathrm{n}) /(\mathrm{n}-1)
$$

onde:

$\lambda=$ a média da medida de consistência de todas as alternativas sob julgamento na matriz

$\mathrm{n}=$ número de alternativas

$$
\text { Razão de Consistência }(C R)=\mathrm{CI} / \mathrm{RI}
$$

onde:

$\mathrm{RI}=$ índice obtido com preenchimento randômico para consistência

A Razão de Consistência (CR) será, então:

$$
C R=\frac{(\lambda-n) /(n-1)}{R I}
$$

O índice RI - Razão de Inconsistência foi simulado por Saaty para matrizes até 9:9, enquanto Forman as estimou até 15:15. Na prática, entretanto, devemos manter o número máximo próximo de 7 , para facilitar a ponderação durante o processo de decisão, conforme mencionamos anteriormente. Os valores para RI são apresentados na Tabela 1 . 
Tabela 1 - Estrutura de comparações de preferências entre pares

\begin{tabular}{cc}
\hline $\boldsymbol{n}$ & $\boldsymbol{R} \boldsymbol{I}$ \\
\hline 2 & 0,00 \\
3 & 0,58 \\
4 & 0,90 \\
5 & 1,12 \\
6 & 1,24 \\
7 & 1,32 \\
8 & 1,41 \\
9 & 1,45 \\
10 & 1,51 \\
\hline
\end{tabular}

O cálculo da Medida de Inconsistência - $\lambda$ é dado pela notação matemática abaixo:

$$
\mathrm{A}=\left(\begin{array}{cccc}
w_{1} / W_{1} & w_{1} / W_{2} & \cdots & w_{1} / W_{n} \\
w_{2} / W_{1} & w_{2} / W_{2} & \cdots & w_{2} / W_{n} \\
\vdots & \vdots & \cdots & \vdots \\
w_{n} / W_{1} & w_{n} / W_{2} & \cdots & w_{2} / W_{n}
\end{array}\right)\left(\begin{array}{c}
P_{1} \\
P_{2} \\
\vdots \\
P_{n}
\end{array}\right)
$$

que, em nosso exemplo, apresenta os seguintes resultados:

\begin{tabular}{|l|c|}
\hline & medida de inconsistência $-\lambda$ \\
\hline Habilidade em Pesquisa e Publicação & 3,150 \\
\hline Visibilidade na Comunidade Acadêmica & 3,082 \\
\hline Titulação & 3,012 \\
\hline
\end{tabular}

resultando uma razão de consistência de:

$$
C R=\frac{(\lambda-n) /(n-1)}{R I}=\frac{(3,081-3) /(3-1)}{0,58}=0,070
$$


Como o valor de CR resultou $0,070<0,1$, temos a consistência das comparações efetuadas nesse exemplo. Caso contrário, a recomendação seria a de proceder à reavaliação das comparações na matriz.

A automação dos procedimentos descritos acima foi facilitada pelo uso de ferramentas de software que podem ser aplicadas, destacando-se o ExpertChoice (EXPERTCHOICE, 2008), ou até mesmo o Excel, que permite que os cálculos sejam feitos em planilha. Abaixo, como exemplo, é fornecida a formulação necessária para que uma matriz de 4:4 seja calculada. Matrizes de outras dimensões podem ser facilmente implementadas alterando-se os parâmetros $n$ e $R I$, na célula I15.

\begin{tabular}{|c|c|c|c|c|c|c|c|c|}
\hline & A & $\mathrm{B}$ & $\mathrm{C}$ & $\mathrm{D}$ & $E$ & $\mathrm{~F}$ & $\mathrm{G}$ & $\mathrm{H}$ \\
\hline 1 & Alternativa & A & B & c & D & & & \\
\hline 2 & A & 1,00 & & & & & & \\
\hline 3 & B & & 1,00 & & & & & \\
\hline 4 & C & & & 1,00 & & & & \\
\hline 5 & D & & & & 1,00 & & & \\
\hline 6 & soma & SOMA(B2:B5) & SOMA(C2:C5) & SOMA(D2:D5) & SOMA(E2:E5) & & & \\
\hline \multicolumn{9}{|c|}{ 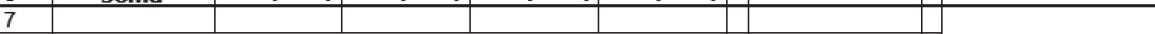 } \\
\hline 8 & \multicolumn{5}{|c|}{ Norma lização } & & & \\
\hline 9 & Alternativa & A & B & C & D & & $\begin{array}{l}\text { PESO DO } \\
\text { ATRIBUTO }\end{array}$ & $\begin{array}{c}\text { medida de } \\
\text { consistência }\end{array}$ \\
\hline 10 & $A$ & $\mathrm{B2} / \mathrm{B} \$ 6$ & $\mathrm{C} 2 / \mathrm{C} \$ 6$ & $\mathrm{D} 2 / \mathrm{D} \$ 6$ & E2/E\$6 & & MÉDIA(B10:E10) & $\begin{array}{c}\text { MATRIZMULT(B2:E2;\$C\$ } \\
\text { 10\$GS13)/G10 }\end{array}$ \\
\hline 11 & B & B3/B\$6 & $\mathrm{C3} / \mathrm{C} \$ 6$ & D3/D\$6 & E3/E\$6 & & MÉDIA(B11:E11) & $\begin{array}{c}\text { MATRIZMULT(B3:E3;\$C\$ } \\
\text { 10\$G\$13)/G11 }\end{array}$ \\
\hline 12 & $\mathrm{C}$ & B4/B\$6 & C4/C\$6 & $D 4 / D \$ 6$ & E4/E\$6 & & MÉDIA(B12:E12) & $\begin{array}{c}\text { MATRIZMULT(BA:E4;\$G\$ } \\
10 \$ G \$ 13) / G 12\end{array}$ \\
\hline 13 & $\mathrm{D}$ & B5/B\$6 & $\mathrm{C} 5 / \mathrm{C} \$ 6$ & D5/D\$6 & E5/E\$6 & & MÉDIA(B13:E13) & $\begin{array}{c}\text { MATRIZMULT(BS:E5;\$C\$ } \$ \\
10 \$ 6 \$ 13) / G 13\end{array}$ \\
\hline \multicolumn{9}{|r|}{ 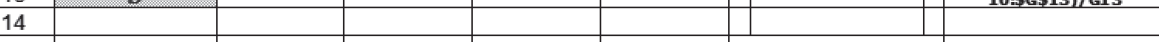 } \\
\hline 15 & & & & & & & $\begin{array}{r}\text { Razão de } \\
\text { Consistência }\end{array}$ & $\begin{array}{c}\text { (MÉDIA(I10:I13)- } \\
4) /\left(3^{*} 0,9\right)\end{array}$ \\
\hline
\end{tabular}

Figura 1 - Programação de AHP para matriz 4:4 em Excel

Fonte: os autores

O Apêndice B apresenta o resultado final do procedimento para determinação dos elementos de credenciamento de docentes do Núcleo Permanente do Programa.

\section{CONCLUSÕES}

Um primeiro resultado deste trabalho foi a própria lista de atributos para o credenciamento de docentes em um Núcleo de Docentes de um Programa de Pós-Graduação e a identificação de seus pesos, que satisfazem a percepção e demanda de um significativo número de interessados na decisão. 
Entretanto, o principal aspecto a ser destacado refere-se à observação de que a aplicação do método desenvolveu em todos os envolvidos o senso de pertença à equipe. Esse sentimento foi percebido como contribuinte para que o grupo alinhasse eventuais diferenças de critérios, percepções e passassem a aceitar o novo conjunto de critérios com os quais não concordaria em um primeiro momento, sem que traços de reações negativas como aversão ao risco ou percepção de perda de autonomia fossem percebidos nos membros da equipe.

Assim, conseguiu-se implantar um esquema de avaliação em uma equipe de elevada autonomia e capacitação, com critérios divergentes das preferências individuais, mas que foram essencialmente aceitos porque a transparência e capacidade de participação foram grandemente enfatizadas, diluindo resistências individuais.

Uma vez utilizada, a técnica permitiu grande transparência do processo e uma maior participação e comprometimento do grupo envolvido. Os resultados foram muito bem assimilados pela equipe, que passou a compreender de maneira mais completa o processo de tomada de decisão, dado à transparência do processo, o que facilitou a gestão do processo como um todo.

Um outro benefício importante observado refere-se à documentação do processo. À medida que os participantes têm dificuldades em lidar com a complexidade de uma decisão que envolve múltiplos aspectos, sua percepção de risco em relação a essa decisão aumenta. Assim, organizar o processo de decisão colegiada torna-se é uma tarefa nada simples, na medida em que múltiplas visões e critérios tendem a confundir o processo de decisão individual, se não houver o suporte de um método que permita a documentação do processo de decisão e sua recuperação a qualquer momento.

Adicionalmente, a capacidade de retomar as etapas parciais de votação individual, permite que a estabilidade do processo, o que torna mais perene a decisão.

\section{REFERÊNCIAS}

BAUMEISTER, R.F.; LEARY, M.R. The need to belong: desire for interpersonal attachments as a fundamental human motivation. Psychological Bulletin, Washington, v. 117, p. 497-529, 1995. 
BENBASAT, I.; GOLDSTEIN, D.K.; MEAD, M. The case research strategy in studies of information systems. MIS Quarterly, Minnesota, v. 11, n. 3, p. 369-386, 1987.

CAPES. Avaliação da pós-graduação. 2008. Disponível em: <http:// www.capes.gov.br/avaliacao/avaliacaopos.html>. Acesso em: 02 abr. 2008.

DE CREMER, D.; BLADER, S. Why do people care about procedural fairness? The importance of belongingness in responding and attending to procedures. European Journal of Social Psychology, United Kingdom, v. 36, n. 2, p. 211-228, 2006.

DIENER, E. Subjective well-being: The science of happiness and a proposal for a national index. American Psychologist, Washington, v. 55, n. 1, p. 34-43, 2000.

EXPERTCHOICE. ExpertChoice 11.5. 2008. Disponível em: < http:// www.expertchoice.com/products/ec11.html $>$. Acesso em: 27 abr. 2008.

FISCHHOFF, B. Value elicitation: is there anything in there? American Psychologist, Washington, v. 46, n.8, p. 835-847, 1991.

FISS, P.C.; ZAJAC, E.J. The symbolic management of strategic change: sensegiving via framing and decoupling. Academy of Management Journal, New York City, v. 49, n. 6, p. 1173-1193, 2006.

GAVIN, J.H.; MASON, R.O. The virtuous organization: The value of happiness in the workplace. Organizational Dynamics, San Francisco, v. 33, n. 4, p. 379-392, 2004.

GIDDENS, A. Modernity and self-identity: self and society in the late modern age. California: Stanford University Press, 1991.

GLIMCHER, P.W.; RUSTICHINI, A. Neuroeconomics: the consilience of brain and decision. Science, Washington, v. 306, n. 5695, p. 447-452, 2004. 
HALLER, M.; HADLER, M. How social relations and structures can produce happiness and unhappiness: An international comparative analysis. Social Indicators Research, New York, v. 75, p. 169-216, 2006.

HARKER, P.T.; VARGAS, L.G. The theory of ratio scale estimation: saaty's analytic hierarchy process. Management Science, Mariland, v. 33, n. 11, p. 1383-1403, nov. 1987.

MILGROM, P.; ROBERTS, J. Economics, organization and management. Upper Side River NJ: Prentice Hall, 1992. 621 p.

MILLER, G.A. The magical number seven, plus or minus two: some limits on our capacity for processing information. The Psychological Review, Washington, v. 63, p. 81-97, 1956.

NG, Y.K. From preference to happiness: towards a more complete welfare economics. Social Choice and Welfare, New York, n. 2, p. $307-3502003$.

PARKER, C.P.; BALTES, B.B.; YOUNG, S.A. et al. Relationships between psychological climate perceptions and work outcomes: A meta-analytic review. Journal of Organizational Behavior, United Kingdom, v. 24, p. 389-416, 2003.

PINDYCK, R.S.; RUBINFELD, D.L. Microeconomia. 6 ed. São Paulo: Prentice Hall, 2006.

SAATY, T.L. A scaling method for priorities in hierarchical structures. Journal of Mathematical Psychology, San Francisco, v. 15, n. 3, p. 234-281, 1977.

. How to make a decision: the Analytic Hierarchy Process. European Journal of Operational Research, San Francisco, v. 48, n. 1, p. 9-26, Sep. 1990.

SPAGNOLO, F.; SOUZA, V. C. O que mudar na avaliação da Capes? Revista Brasileira de Pós-Graduação, Brasilia, v. 1, n. 2, p. 8-34, 2004. 
SUSMAN, G.I.; EVERED, R.D. An assessment of the scientific merits of action research. Administrative Science Quarterly, New York, v. 23, p. 582-603, 1978.

THIOLLENT, M. Pesquisa-Ação nas Organizações. São Paulo: Atlas, 1997.

ZAHEDI, F. Group consensus function estimation when preferences are uncertain. Operations Research, Mariland, v. 34, n. 6, p. 883894, Nov-Dec, 1986. 


\section{APÊNDICE A - HIERARQUIA INICIAL OBTIDA PELO PROCESSO DE BRAIN-STORMING}

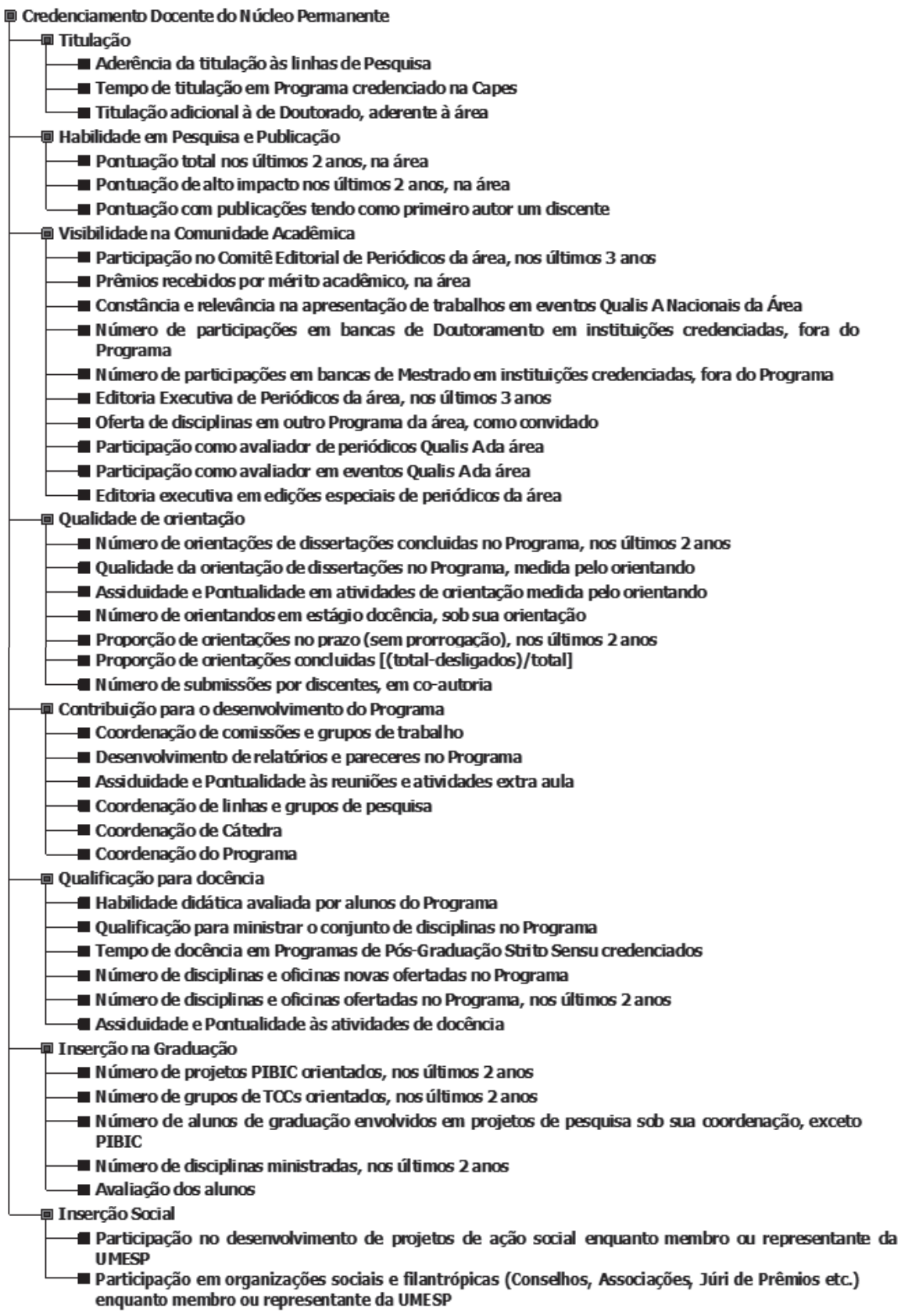




\section{APÊNDICE B - RESULTADO FINAL DOS PESOS DOS ATRIBUTOS PARA CREDENCIAMENTO DE DOCENTES DO NÚCLEO PERMANENTE}

Habilidade em Pesquisa e Publicação (na área) (0,261)

Pontuação de alto impacto $(0,673)$

Pontuação de produção intelectual $(0,176)$

Pontuação com publicações tendo como primeiro autor um discente $(0,152)$

Qualidade de orientação $(0,149)$

Qualidade das orientações, avaliada pelos orientandos $(0,341)$

Número de submissões em co-autoria com discentes $(0,228)$

Histórico de orientações no prazo (sem prorrogação) $(0,152)$

Assiduidade e Pontualidade em atividades de orientação $(0,151)$

Número de orientações de dissertações concluídas $(0,127)$

Visibilidade na Comunidade Acadêmica (da área) (0,149)

Atuação como avaliador de periódicos Qualis A $(0,199)$

Editoria de periódicos $(0,186)$

Constância na apresentação de trabalhos em eventos Qualis A $(0,162)$

Participações em bancas de Doutorado, fora do Programa $(0,113)$

Prêmios recebidos por mérito acadêmico $(0,112)$

Atuação como avaliador em eventos Qualis A $(0,108)$

Participações em bancas de Mestrado, fora do Programa $(0,072)$

Disciplinas ministradas em outro Programa $(0,048)$

\section{Contribuição para o desenvolvimento do Programa $(0,148)$}

Coordenação do Programa $(0,363)$

Coordenação de linhas de pesquisa $(0,223)$

Coordenação de comissões e grupos de trabalho $(0,163)$

Assiduidade e Pontualidade às reuniões e atividades extra aula $(0,097)$

Preparação de relatórios e pareceres $(0,081)$

Coordenação de Cátedra $(0,073)$

\section{Qualificação para docência no Programa $(0,119)$}

Qualificação para ministrar o conjunto de disciplinas no Programa $(0,293)$

Habilidade didática, avaliada por alunos $(0,269)$

Histórico de disciplinas e oficinas ofertadas no Programa $(0,167)$

Assiduidade e Pontualidade às atividades de docência $(0,140)$

Tempo de docência em Programas Strito Sensu $(0,132)$ 
Titulação (aderente à área) $(\mathbf{0 , 0 8 7 )}$

Titulação adicional à de Doutorado $(0,448)$

Aderência da titulação à área $(0,404)$

Tempo de titulação $(0,148)$

Inserção Social (enquanto membro ou representante da Instituição) $(0,048)$

Participação em projetos de ação social $(0,523)$

Participação em organizações sociais e filantrópicas (Conselhos, Associações, Júri de Prêmios etc.) $(0,477)$

Inserção na Graduação (0,040)

Orientações de projetos $\operatorname{PIBIC}(0,310)$

Orientações em projetos de pesquisa, exceto $\operatorname{PIBIC}(0,243)$

Orientações de TCCs $(0,217)$

Qualidade de aulas na graduação, avaliada pelos alunos $(0,155)$

Quantidade de disciplinas ministradas $(0,074)$ 